\title{
Uniportal versus multiportal thoracoscopic sleeve lobectomy for the surgical treatment of centrally located lung cancer: a single institution experience
}

\author{
Jun Zhao", Qingpeng Zeng"^ ${ }^{\#}$, Jiagen Li, Fengwei Tan, Qi Xue, Juwei Mu, Yushun Gao, Dali Wang, \\ Shugeng Gao
}

Department of Thoracic Surgery, National Cancer Center/National Clinical Research Center for Cancer/Cancer Hospital, Chinese Academy of Medical Sciences and Peking Union Medical College, Beijing, China

Contributions: (I) Conception and design: J Zhao, S Gao; (II) Administrative support: S Gao; (III) Provision of study materials or patients: Q Xue, J Mu, Y Gao, D Wang; (IV) Collection and assembly of data: Q Zeng; (V) Data analysis and interpretation: J Zhao, Q Zeng, J Li, F Tan; (VI) Manuscript writing: All authors; (VII) Final approval of manuscript: All authors.

\#These authors contributed equally to this work.

Correspondence to: Shugeng Gao, MD. Department of Thoracic Surgery, National Cancer Center/National Clinical Research Center for Cancer/ Cancer Hospital, Chinese Academy of Medical Sciences and Peking Union Medical College, No. 17, Panjiayuan Nanli, Beijing 100021, China. Email: gaoshugeng@vip.sina.com.

Background: Uniportal thoracoscopic sleeve lobectomy is rarely reported owing to its high degree of difficulty. We conducted a comparative study on the safety and efficacy of uniportal versus multiportal thoracoscopic sleeve lobectomy for the treatment of centrally located lung cancer.

Methods: From January 2016 to December 2018, 30 thoracoscopic sleeve lobectomies (12 by the uniportal approach and 20 by the multiportal approach) for centrally located lung cancer at our institution were retrospectively analyzed.

Results: The uniportal approach resulted in a significantly shorter chest drainage duration $(5.3 \pm 1.9$ vs. $7.1 \pm 2.8$ days, $\mathrm{P}=0.028)$ and a smaller chest drainage volume $(796.7 \pm 582.9$ vs. $1,667.8 \pm 1,154.9 \mathrm{~mL}, \mathrm{P}=0.004)$ than the multiportal approach. The two groups showed no significant differences in the dissection of lymph nodes, operation time, estimated blood loss, conversion rate, length of postoperative hospital stay and the proportion of patients with postoperative complications. The short-term overall survival (OS) and diseasefree survival (DFS) between uniportal and multiportal groups were similar (3-year OS, $100.0 \%$ vs. $82.5 \%$, $\mathrm{P}=0.222$; 3-year DFS, $75.8 \%$ vs. 84.4\%, $\mathrm{P}=0.641$ ). For the eight cases of the uniportal approach conducted by the same surgeon, the cumulative sum (CUSUM) curve showed its inflection at patient number 4 and divided the series into phase I (learning phase) and phase II (experienced phase). A significant reduction in estimated blood loss $(42.5 \pm 8.7$ vs. $177.5 \pm 121.2 \mathrm{~mL}, \mathrm{P}=0.037)$, chest drainage volume $(280.0 \pm 155.8$ vs. $972.5 \pm 464.5 \mathrm{~mL}, \mathrm{P}=0.043)$ and chest drainage duration $(3.8 \pm 1.0$ vs. $6.8 \pm 2.2$ days, $\mathrm{P}=0.027)$ was also noted in the phase II patients compared with the phase I patients.

Conclusions: Uniportal thoracoscopic sleeve lobectomy is technically feasible and safe for the treatment of centrally located lung cancer and may achieve superior surgical outcomes compared with the multiportal approach.

Keywords: Uniportal thoracoscopic surgery; multiportal; sleeve lobectomy; lung cancer

Submitted Aug 19, 2020. Accepted for publication Oct 22, 2020.

doi: $10.21037 /$ jtd-20-2695

View this article at: http://dx.doi.org/10.21037/jtd-20-2695

^ ORCID: 0000-0002-7891-8271. 


\section{Introduction}

Bronchial sleeve lobectomy is an optional and effective procedure for resectable centrally located lung cancer. A previous study showed that sleeve lobectomy avoids the residual risk of tumors caused by anatomical lobectomy and offers a better prognosis with similar morbidity and mortality in selected patients (1). Owing to its complexity and high risk of complications, thoracotomy has always been the standard approach for sleeve lobectomy. In recent decades, with the development of thoracoscopic technology and the accumulation of surgical techniques, some surgeons have begun to apply thoracoscopic technology for sleeve lobectomy. Since Santambrogio et al. (2) reported the first case of thoracoscopic sleeve lobectomy in 2002, several studies have verified the safety and efficacy of thoracoscopic sleeve lobectomy $(3,4)$; however, all these cases have been conventionally performed with three or four incisions (1 observation port plus 2-3 operation ports).

The first uniportal thoracoscopic lung lobectomy was performed by Gonzalez et al. (5) in 2011, and since then, the uniportal video-assisted thoracoscopic surgery (VATS) has been gradually applied to almost all lung cancer resections and has shown advantages in minimizing injury and postoperative pain. In 2013, Gonzalez-Rivas et al. (6) reported the first uniportal thoracoscopic sleeve lobectomy. Subsequently, several studies have described uniportal thoracoscopic sleeve lobectomy (7-10) and the even more complex double sleeve lobectomy (11). With the accumulation of VATS techniques in our institute, we have gradually applied the advantages of VATS to patients who required sleeve lobectomies and performed the first uniportal thoracoscopic sleeve lobectomy in January 2016. However, uniportal thoracoscopic sleeve lobectomy remains a technically demanding operation, and most sleeve lobectomy still needs to be performed by conventional thoracoscopic or thoracotomy procedures, even for the most experienced thoracic surgeons. To date, all published data has been limited in the form of case reports, case series or uncontrolled observational studies $(11,12)$. Therefore, we conducted this comparative study to explore the safety and efficacy of uniportal thoracoscopic sleeve lobectomy for the treatment of centrally located lung cancer and present our current experiences, which might provide more guidance for surgeons dealing with related issues. We present the following article in accordance with the STROBE reporting checklist (available at http://dx.doi.org/10.21037/jtd-202695).

\section{Methods}

\section{Patient enrollment}

We retrospectively reviewed all patients who underwent thoracoscopic sleeve lobectomy for centrally located lung tumors from January 2016 to December 2018. The patients were categorized into uniportal and multiportal groups. For centrally located lung cancer, the indication for a thoracoscopic sleeve lobectomy includes restricted bronchus invasion, no signs of vessel or surrounding organ invasion, no mediastinal lymph node or distant metastasis and pulmonary function being able to tolerate the planned lobectomy or potential pneumonectomy. For a better comparison between the uniportal and multiportal approaches, we excluded patients who received preoperative treatment (including chemotherapy and/or radiotherapy) to reduce the potential bias. This study was conducted in accordance with the Declaration of Helsinki (as revised in 2013). Study had been approved by the Research Ethics Committee of Cancer Institute and Hospital of the Chinese Academy of Medical Sciences (No. 19-085-1870) and informed consent was taken from all the patients.

\section{Operative procedure}

All operations were performed by a total of six surgeons whose annual operating volume surpassed 200 cases in our department. Nearly all of the surgeries were performed via uniportal approach throughout the study period. For sleeve lobectomy, the decision to perform a uniportal or multiportal approach depended on the discussion of the multiple disciplinary team (MDT). The incisions for a conventional multiportal approach have been described in previous literature (13). For the uniportal approach, a single $3-4 \mathrm{~cm}$ incision was made in the 4 th or 5 th intercostal space at the anterior axillary line according to the patient's body conformation and resection of the lobe. The surgeon stood on the ventral side of the patient, and the camera man stood on the contralateral or ipsilateral side according to upper lobectomy or lower lobectomy. The camera was placed at the dorsal side of the incision, and the remaining instruments entered the incision from the ventral side of the lens.

No extensive pleural adhesion and metastasis were found in any patient through thoracoscopic exploration. The bronchus was identified and dissected sharply from the initial part of the bronchus by a scalpel with a long 
handle, and then the scissor was used to transect the remaining circumference of the bronchus. Electrocautery and an ultrasonic knife were not recommended for the possibility of causing stump necrosis. Both the proximal and distal bronchial margins were then sent as frozen sections to confirm negative margins, after which systematic mediastinal lymph node dissection was performed before anastomosis to provide greater maneuvering space and save time. The details of the lymphadenectomy were outlined in a prior publication (14). For sleeve resection of the upper or middle lobe, the inferior pulmonary ligament was first loosened before anastomosis for further mobilization of the lower or middle-lower lobe and a reduction in the anastomotic tension. For sleeve resection of the right lobes, the azygos vein arch could be resected according to the actual need of the operation. All the patients underwent an end-to-end bronchial anastomosis with continuous suturing using a single 3-0 Prolene suture (Ethicon Inc., Somerville, NJ, USA). The anastomosis technique used in the uniportal approach was similar to that in the multiportal approach, which was reported in a recent publication (15).

\section{Postoperative management}

Patients were regularly managed in the dedicated thoracic surgical ward or transferred into the intensive care unit (ICU) postoperatively for the management of severe complications. Standard postoperative care also included atomization inhalation, patients' early mobilization, assistance of sputum clearance and pulmonary re-expansion. A daily chest drainage volume less than $200 \mathrm{~mL}$ without obvious air leakage indicated that the chest tube could be removed.

\section{Data collection}

Patient demographic and clinical features were collected and analyzed. The pathologic stages were based on the 8th TNM classification system (16). Perioperative mortality was defined as any patient who died within the first 30 days postoperatively. The follow-up information was obtained by contacting patients or requesting the information from our follow-up center. Overall survival (OS) was calculated from the date of surgery to the date of death by any cause or last follow-up. Disease-free survival (DFS) was calculated from the date of surgery to the date of the first identified recurrence, death by any cause, or last follow-up.

\section{Statistical analysis}

Statistical analyses were performed using SPSS (IBM, Version 23.0, Armonk, NY, USA). Figures were generated using GraphPad Prism (GraphPad Software Inc., version 8.0, La Jolla, CA, USA). Continuous variables are presented as the mean \pm standard deviation $(\mathrm{SD})$ and were compared using the Wilcoxon rank-sum test. Categorical variables are presented as frequencies and percentages and compared using the chi-square test or Fisher's exact test. Survival outcomes were calculated using the Kaplan-Meier estimators and compared using the log-rank test. The Pearson correlation coefficient was applied to analyze the correlation between the consecutive number of cases and operation time. The cumulative sum (CUSUM) method was used to assess the learning curve using the cumulative sum value of operation time $\left(\mathrm{CUSUM}_{\mathrm{OT}}\right)$, which was described previously by Zhang et al. (17). A P value less than 0.05 was considered to be statistically significant.

\section{Results}

\section{Demographic features}

A total of 40 patients who underwent thoracoscopic sleeve lobectomy were identified, and eight cases were excluded from the study for receiving preoperative treatment (Figure 1). In this analysis cohort, 12 patients received the uniportal approach, and 20 patients received the multiportal approach. The patient characteristics of the uniportal and multiportal groups are described in Table 1. Male patients were predominant in both groups, and the median age of all patients was 60.5 years. The two groups showed no significant differences in demographic variables, which indicated that the baseline data between the two groups were comparable.

\section{Perioperative results}

Table 2 shows the perioperative results for the two groups. In terms of tumor characteristics, there was a similar tumor size, histology and pathologic stage between the two groups. The most common procedure in all patients was right upper lobe (RUL) sleeve resection, followed by left upper lobe (LUL) sleeve resection. The mean number of stations and number of lymph node dissections were similar between the two groups. All the patients had achieved a complete $\mathrm{R} 0$ resection, and no transfusion was required throughout the hospital stay. One patient $(8.3 \%)$ in the uniportal 


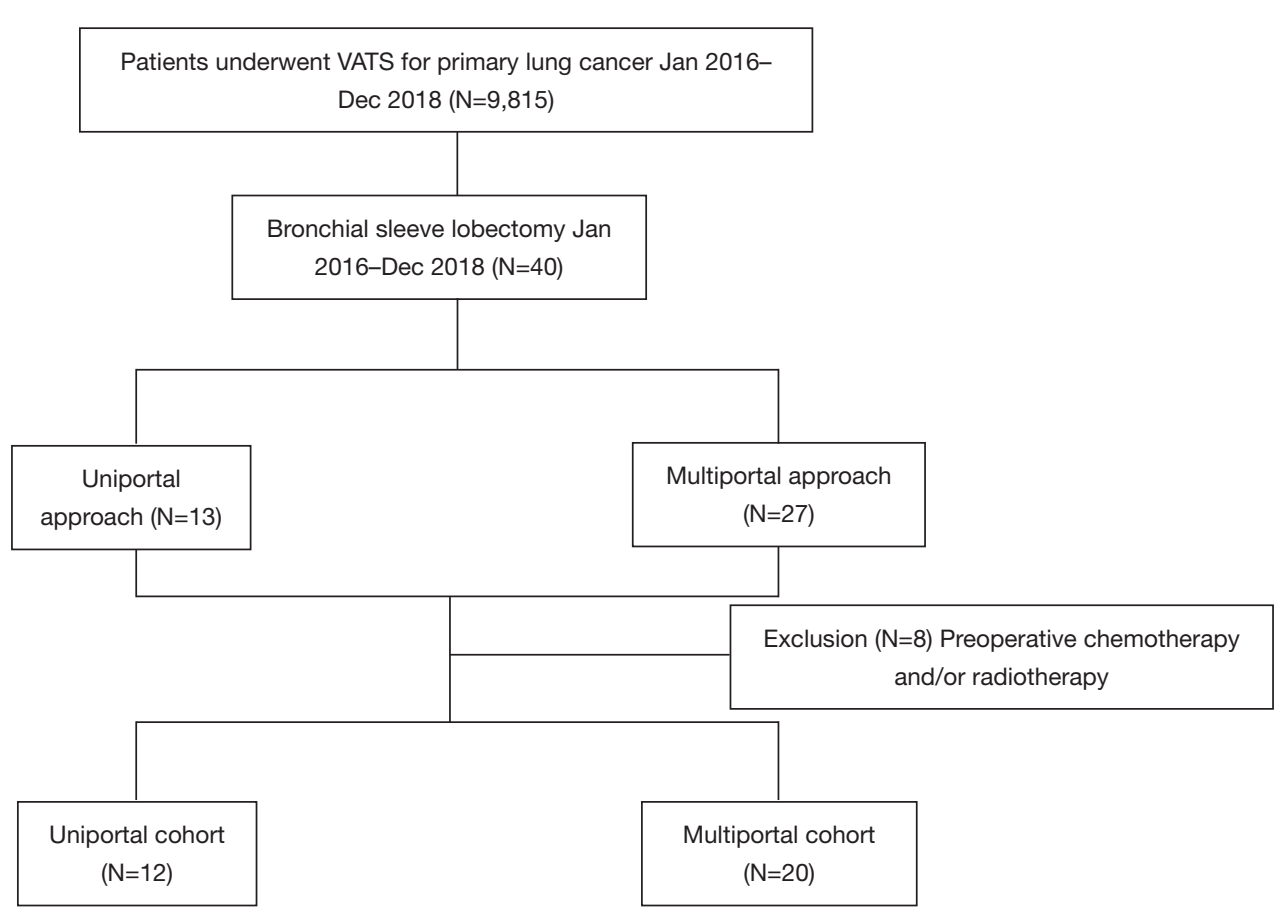

Figure 1 Flowchart for patient inclusion and exclusion. VATS, video-assisted thoracoscopic surgery.

Table 1 Baseline characteristics between the uniportal and multiportal groups

\begin{tabular}{|c|c|c|c|}
\hline Characteristics & Uniportal $(n=12)$ & Multiportal $(n=20)$ & $P$ value ${ }^{a}$ \\
\hline Age, y & $56.2 \pm 14.2$ & $61.0 \pm 8.0$ & 0.471 \\
\hline $\mathrm{BMI}, \mathrm{kg} / \mathrm{m}^{2}$ & $26.2 \pm 4.6$ & $24.0 \pm 3.0$ & 0.227 \\
\hline Smoking history & $6(50.0)$ & $16(80.0)$ & 0.119 \\
\hline FVC/predicted & $79.1 \pm 17.6$ & $78.5 \pm 14.5$ & 0.586 \\
\hline $\mathrm{FEV}_{1} /$ predicted & $77.2 \pm 17.8$ & $77.2 \pm 16.4$ & 0.938 \\
\hline Comorbidities $^{b}$ & & & 0.840 \\
\hline Coronary artery disease & $1(8.3)$ & $3(15.0)$ & \\
\hline Hypertension & $2(24.1)$ & $4(20.0)$ & \\
\hline
\end{tabular}

Variables are presented as the mean $\pm \mathrm{SD}$ or $\mathrm{n}(\%) .^{\text {a }}$, based on Wilcoxon rank-sum or Fisher exact tests; ${ }^{\mathrm{b}}$, one patient may have one

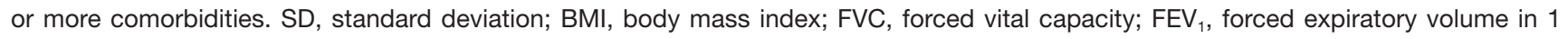
second; COPD, chronic obstructive pulmonary disease. 
Table 2 Tumor characteristics and operative details

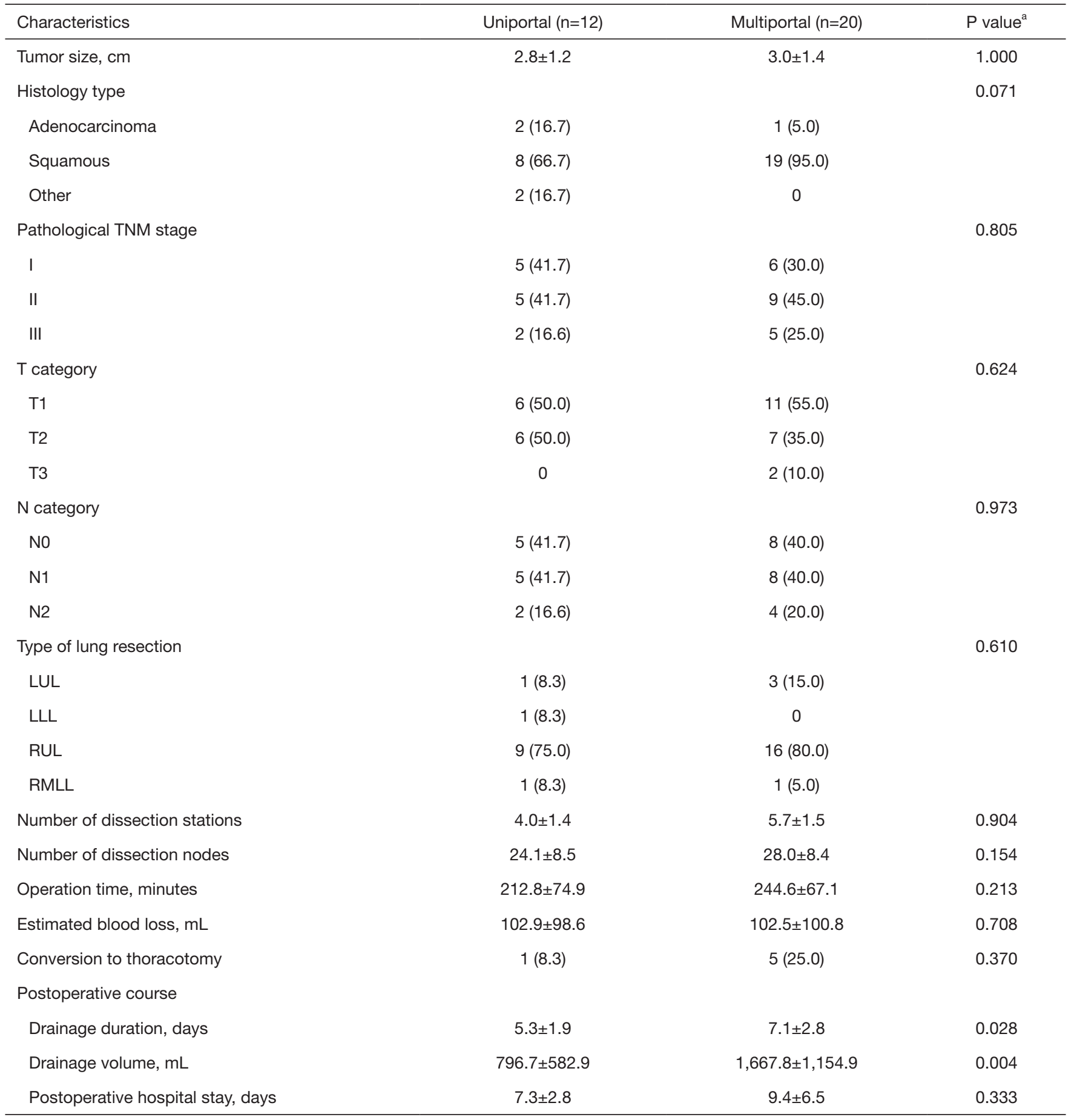

Variables are presented as the mean \pm SD or $\mathrm{n}(\%) .{ }^{a}$, based on Wilcoxon rank-sum or Fisher exact tests. SD, standard deviation; LUL, left upper lobe; LLL, left lower lobe; RUL, right upper lobe; RMLL, right middle and lower lobe. 
Table 3 Hospital course, morbidity and postoperative treatment

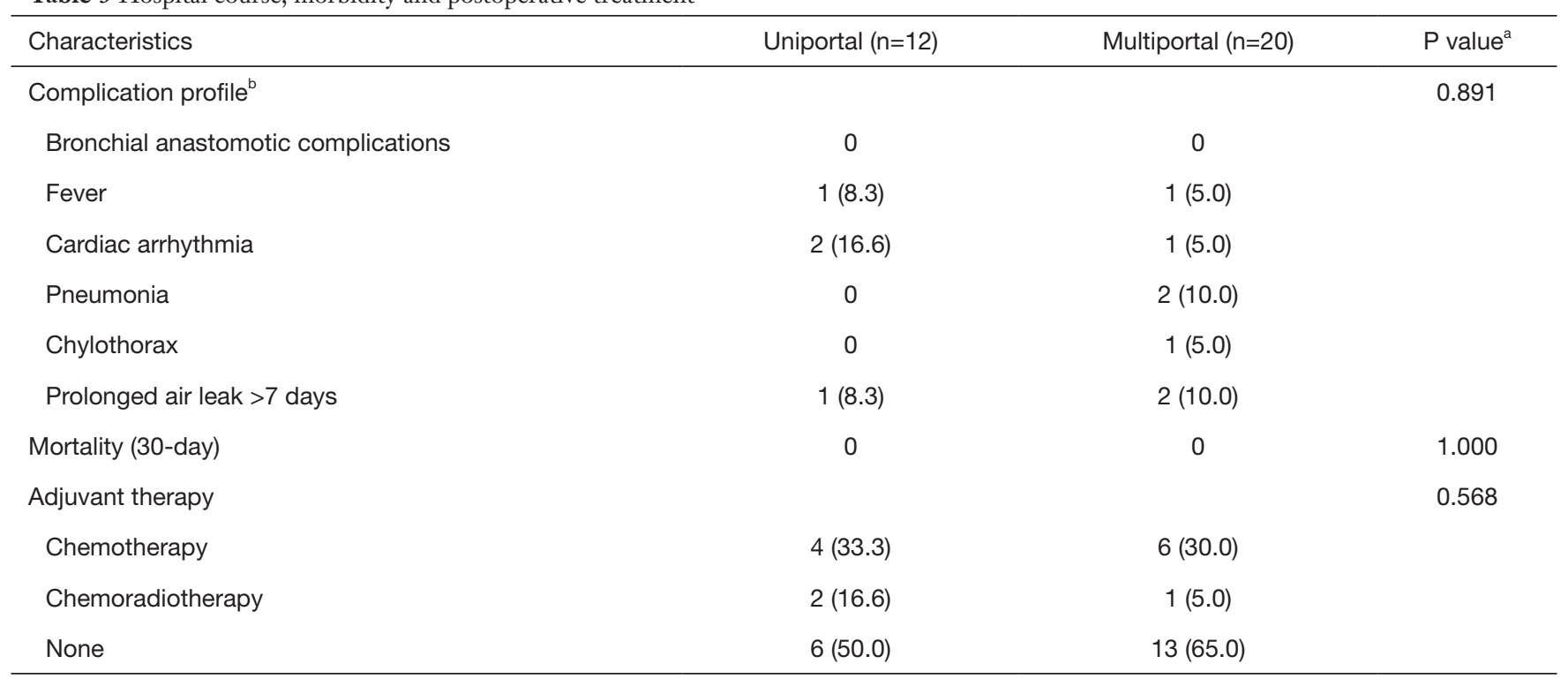

Variables are presented as $\mathrm{n}(\%){ }^{a}$, based on the Fisher exact test; ${ }^{\mathrm{b}}$, one patient may experience several complications.

group and $5(25 \%)$ in the multiportal group experienced intraoperative conversion to thoracotomy; however, this difference did not reach the significance level $(\mathrm{P}=0.370)$. In addition, the two groups showed no significant differences in terms of the operation time, estimated blood loss, and the length of postoperative hospital stay. It is notable that the uniportal approach, compared to the multiportal approach, resulted in a significantly smaller mean volume of chest drainage $(796.7 \pm 582.9$ vs. $1,667.8 \pm 1,154.9 \mathrm{~mL}$, respectively; $\mathrm{P}=0.004)$ and a shorter mean chest drainage duration (5.3 \pm 1.9 vs. $7.1 \pm 2.8$ days, respectively; $\mathrm{P}=0.028$ ). As shown in Table 3, 4 patients $(33.3 \%)$ in the uniportal group and 7 (35.0\%) in the multiportal group experienced postoperative complications. The proportion of patients with complications in the two groups showed no significant difference $(\mathrm{P}=0.891)$. There was no perioperative death in either group. Six patients $(50.0 \%)$ in the uniportal group and $7(35.0 \%)$ in the multiportal group received adjuvant chemotherapy or chemoradiotherapy, but no significant difference was observed $(\mathrm{P}=0.568)$.

\section{Follow-up information}

All patients $(\mathrm{n}=32)$ completed follow-up, and the median follow-up time was 29.0 (range, 7-45) months. No death event was observed and two patients experienced relapse in the uniportal group. In the multiportal group, three patients deceased during the follow-up. The 3-year OS and DFS rates were $100.0 \%$ and $75.8 \%$ for uniportal group, $82.5 \%$ and $84.4 \%$ for multiportal approach. Figure 2 shows the survival curves for OS and DFS between the two groups. No significant differences were observed between the two groups either in OS $(\mathrm{P}=0.222)$ or in DFS $(\mathrm{P}=0.641)$.

\section{Learning curve analysis}

Of the 12 uniportal cases, eight operations were conducted by the same surgeon (Dr. JZ). To demonstrate a specific learning curve for the uniportal approach in the treatment of centrally located lung cancer, we chose these eight uniportal cases to construct the curve. In our experience, there was a significant inverse linear correlation between the surgical cases and operation time (Pearson's $r=-0.907, \mathrm{P}=0.002$ ), which also indicated that after four cases, the operative time was shorter than the mean operative time $(223 \mathrm{~min})$ of all uniportal series (Figure 3). The CUSUM $\mathrm{OT}_{\mathrm{OT}}$ curve was plotted in chronological case order, and a four-case cut-off point was established based on the consistent inflection in the curve (Figure 4). The series was then divided into phase I (the first our cases) and phase II (the last four cases), which separately represented the learning phase (ascending slope) and the experienced phase (descending slope). As shown in Figure 5, the perioperative parameters were compared between the two phases, and a significant reduction in estimated blood loss $(42.5 \pm 8.7$ vs. $177.5 \pm 121.2 \mathrm{~mL}$, $\mathrm{P}=0.037)$, chest drainage volume $(280.0 \pm 155.8$ vs. 
A

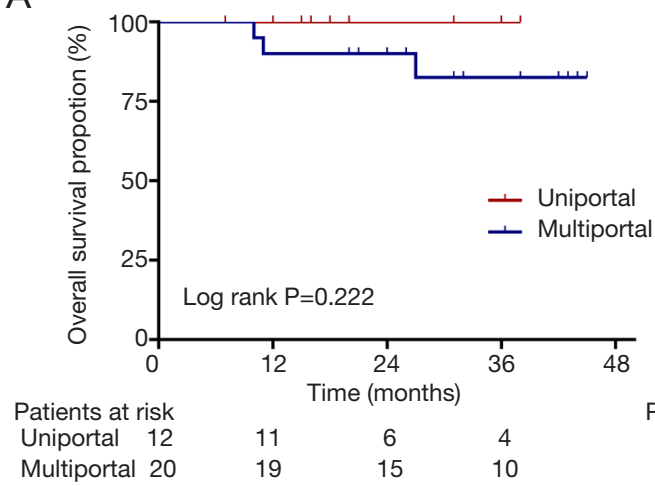

B

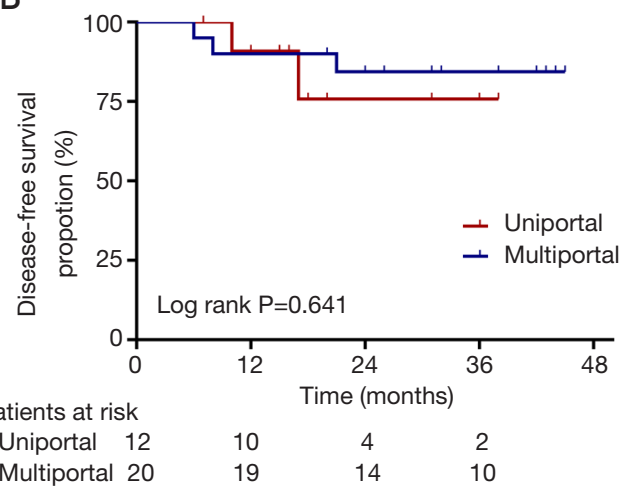

Figure 2 Kaplan-Meier curve for OS (A) and DFS (B) by the uniportal and multiportal approach. OS, overall survival; DFS, disease-free survival.

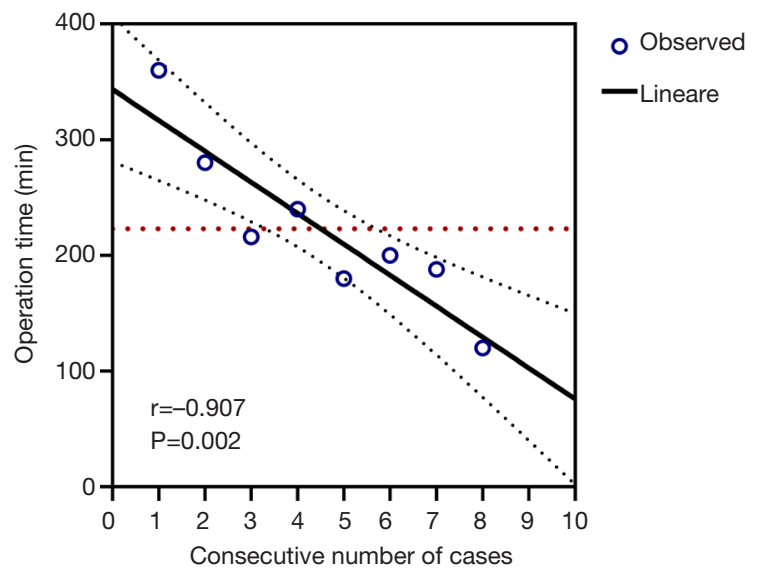

Figure 3 Linear regression analysis between the consecutive number of cases and operation time (Pearson's $\mathrm{r}=-0.907, \mathrm{P}=0.002$ ). The red line shows the average operation time for the uniportal approach (223 $\mathrm{min})$.

$972.5 \pm 464.5 \mathrm{~mL}, \mathrm{P}=0.043)$ and chest drainage duration ( $3.8 \pm 1.0$ vs. $6.8 \pm 2.2$ days, $\mathrm{P}=0.027)$ was noted in phase II patients compared with phase I patients, respectively. However, no significant difference was observed in the postoperative hospital stay between phase I and phase II $(6.8 \pm 2.9$ vs. $7.0 \pm 2.2$ days, respectively; $\mathrm{P}=0.882)$.

\section{Discussion}

This current study suggests that uniportal thoracoscopic surgery is a safe and feasible approach for pulmonary sleeve lobectomy and provides a superior perioperative result compared with a multiportal approach. In addition, the early learning curve of this complex procedure shows that a relatively short period was required to reach a performance plateau for surgeons with extensive uniportal VATS experience.

In terms of surgical outcomes, the uniportal approach showed no inferiority to the multiportal approach in terms of operation time, R0-resection, lymph node dissection, and conversion rate. Several publications have reported similar conclusions $(14,18)$. Notably, the uniportal approach provided obvious advantages in aspects of chest drainage duration and volume compared to the multiportal approach. The decreased surgical incisions may be one of the reasons contributed to this phenomenon, however, this assumption still need verification in large prospective studies.

Although the difference did not reach the significance level, the uniportal group showed a trend toward a shorter postoperative hospital stay. This altogether contributes to patients' relief of postoperative pain and financial burden.

Bronchial anastomotic complications such as bronchopleural fistula or anastomotic stricture are surgeons' major concerns for sleeve lobectomy. In our study, no such complication was observed in either group. In the previously published literature, including a retrospective cohort of eight uniportal sleeve lobectomies conducted by Chen (14) and an observational prospective cohort of 38 uniportal sleeve lobectomies conducted by Koryllos (18), no anastomotic complications were observed. These findings suggest that uniportal thoracoscopic sleeve lobectomy, although more surgically complicated, could also be performed with no increased risk of postoperative complications. However, patient selection bias and limited case series may also lead to this result. 


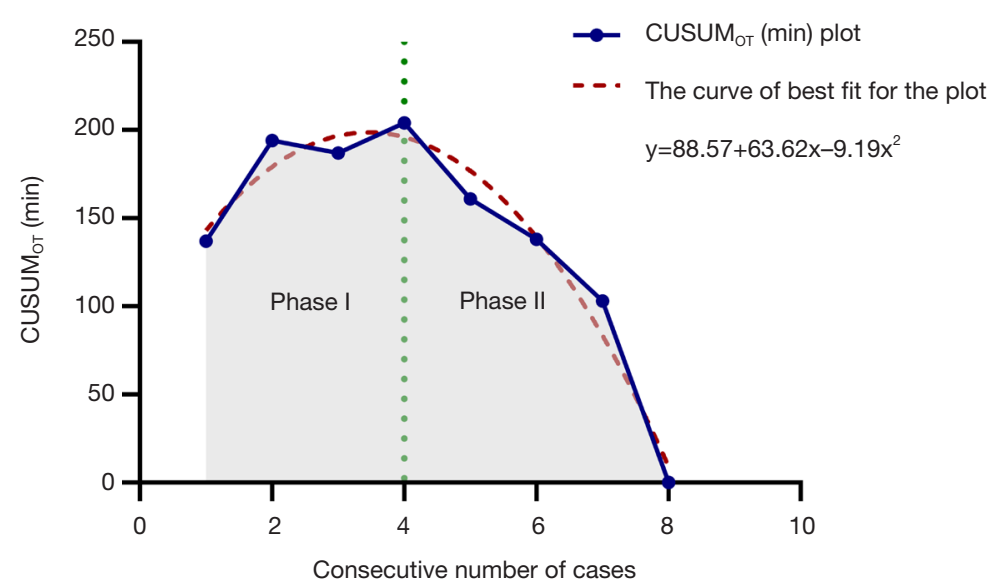

Figure 4 CUSUM $_{\text {От }}$ plot for uniportal thoracoscopic sleeve lobectomy (blue line). The red dashed line represents the curve of best fit for the plot (a second-order polynomial with the equation CUSUM $_{\mathrm{OT}}=88.57+63.62 \times$ case number $-9.19 \times$ case number $^{2}$ ). The first four patients comprised the ascending slope of the curve (phase I), and the last four patients comprised the descending slope of the curve (phase II). CUSUM $\mathrm{OT}_{\mathrm{OT}}$, cumulative sum value of operation time.

To date, no study has reported survival outcomes after uniportal sleeve lobectomy. In our study, although the 5-year survival rate could not be compared since most uniportal cases were performed in recent years, the short-term OS or DFS between the two groups was similar, which suggests that the uniportal procedure causes no impairment to the prognosis for sleeve lobectomy patients.

Previous concerns about uniportal sleeve lobectomy have focused on a complete oncologic resection and a safe anastomosis. Through this study, we are confident in achieving comparable or even better outcomes with a uniportal approach. However, these results are mostly attributable to experience accumulation from conventional multiportal lobectomies. Preoperative patient selection is also a crucial aspect for uniportal thoracoscopic sleeve lobectomy, and rigorous screening and preoperative evaluation based on enhanced computed tomography (CT) scans or bronchoscopy are needed. For patients with a large tumor, the close relationship between the tumor and hilar vessels and multiple enlarged lymph nodes in the hilum and mediastinum suggests that the choice of a uniportal approach should be carefully assessed or submitted to an MDT for discussion. Moreover, in our study, most of the uniportal cases (nine patients) underwent sleeve resection of the RUL. The reason lies in the high incidence of malignancies in the RUL of the lung; on the other hand, sleeve resection of the RUL is technically easier than that of the other lobes. Sleeve resection of the LUL shows additional complexity from interference of the aortic arch and the frequent variation in the pulmonary artery. These findings indicate that cautious and strict selection of cases for a uniportal sleeve lobotomy is equally important.

The uniportal thoracoscopic approach provides a direct vision and a similar surgical approach as the thoracotomy approach; however, it undoubtedly restricts the field of vision and may cause mutual interference among surgical instruments. In our experience, the location of the incision needs comprehensive consideration according to the patient's body conformation, resection of the lobe and operators' habits. For the upper sleeve lobectomy, the incision was mostly taken at the 5 th intercostal space of the axillary midline; for the lower or middle-lower sleeve lobectomy, the 4th intercostal space of the axillary midline would be more feasible. For patients with a wide anteroposterior thoracic diameter, the incision should be properly moved backward, and vice versa. For the upper sleeve lobectomy, the camera assistant stands on the contralateral side to avoid interference with the surgeon's manipulation. For the lower or middle-lower sleeve lobectomy, the camera assistant stands on the ipsilateral and cranial side of the surgeon, thus eliminating incongruous reverse-operational vision. During the operation, the thoracoscopic lens should be placed as flat as possible to provide more space for the surgeon's manipulation. In terms of anastomosis, Mahtabifard et al. (4) recommend interrupted sutures for reliable anastomosis, better size matching and less loosening or entanglement of the sutures. However, several knots would be placed in the bronchial 
A

C
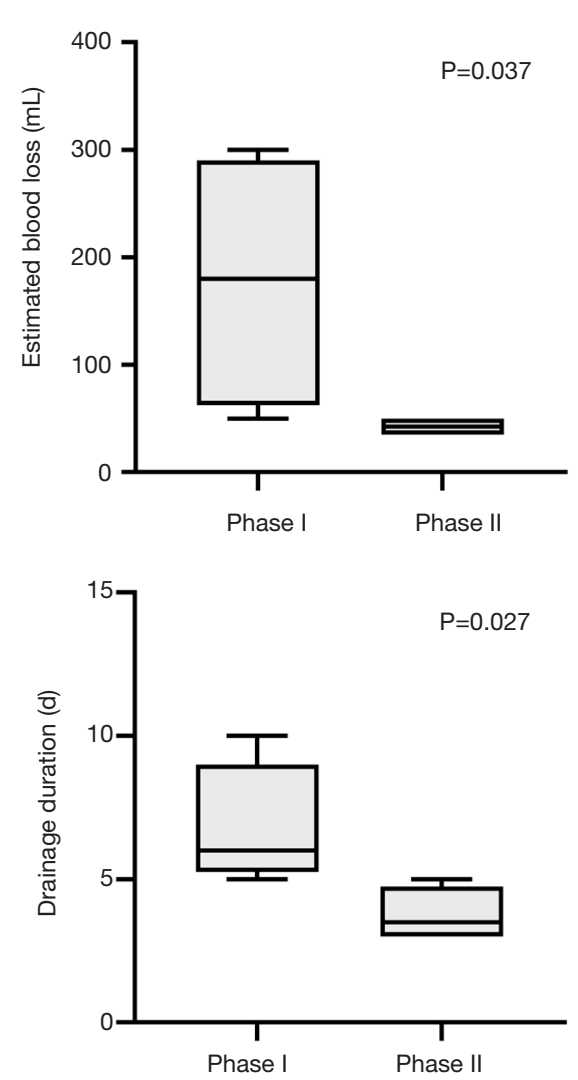

B
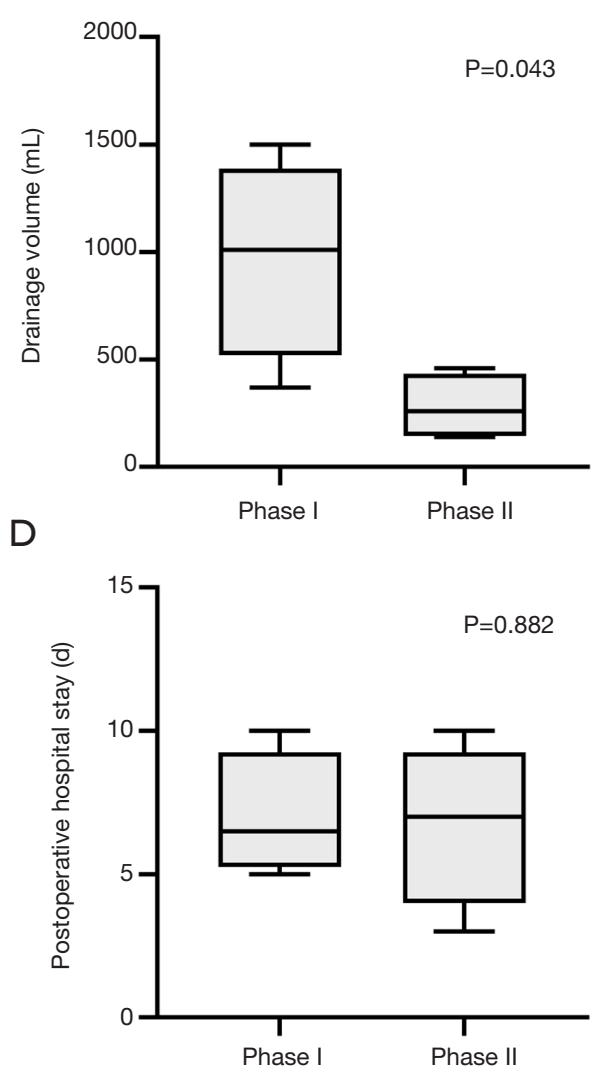

Figure 5 Interphase comparison of the perioperative outcomes. (A) Estimated blood loss; (B) chest drainage volume; (C) chest drainage duration; (D) postoperative hospital stay.

lumen, which may cause postoperative sputum retention, irritable cough and even anastomotic stricture (13). Some authors combined continuous and interrupted suturing for the membranous and cartilaginous portions, respectively, to offset the limitations of simple interrupted sutures $(13,19)$. In our patients, we applied complete continuous suture throughout the anastomosis and achieved satisfying results. Similar conclusions were also obtained from Chen's study (14). Hence, we assumed that without a single knot placed in the bronchial lumen, this kind of anastomosis may be applied without causing additional postoperative symptoms and anastomotic stricture. In addition to easier manipulation, this technique could also largely reduce the anastomosis time. Another crucial aspect of this technique is the management of the sutures to prevent tangling. For continuous anastomosis, one-site interruption of the suture or twist of the thread would result in an anastomosis failure. At this point, the importance of the second assistant appears particularly prominent. The polypropylene suture is double- ended with two needles. During anastomosis, the idle end was fixed on one side of the incision, which was opposite the anastomotic direction, or wrapped around a gauze roll and placed in the thoracic cavity distant to the stump of the trachea to be anastomosed. The other end used for anastomosis was also fixed on the same side of the incision by the assistant, thus making the intrathoracic sutures in a straightening state, which can effectively avoid the twist of the sutures, increase the success rate of anastomosis and shorten the operation time.

The CUSUM method has been commonly used to evaluate a surgeon's initial and ongoing performance of procedures. Although the operation time varied according to the complexity of different individuals, our plots present with a gradual decline in operation time. Unlike other previous studies concerning CUSUM analysis, we failed to reach a clear plateau phase of the learning curve due to the limited number of cases; however, phase I (learning phase) and phase II (experienced phase) were clearly differentiated. 
After crossing the learning phase, we also observed significantly less estimated blood loss and chest drainage volume and shorter chest drainage stay, which indicated that careful maneuvering and less operative trauma were particularly required in the learning phases. Undoubtedly, the learning curve may vary in different centers, and the relatively short period in our institution may be attributed to the previous accumulation of experience.

To our knowledge, this is the first study to compare a uniportal and multiportal approach for the thoracoscopic sleeve lobectomy of centrally located lung cancer. However, some limitations in this study still exist. First, this is a nonrandomized and retrospective single-institution study that might have resulted in a certain degree of bias. Second, there were only 32 cases included in this study due to the technical complexity of thoracoscopic sleeve lobectomy. The small sample size may have reduced the power of the statistical tests. Third, even though we excluded patients who underwent preoperative chemotherapy or chemoradiotherapy, unknown confounding variables and inequalities between the two groups could have biased the results. Moreover, the limited follow-up period may have also led to premature conclusions.

\section{Conclusions}

Despite a small sample size, we can preliminarily conclude that uniportal thoracoscopic sleeve lobectomy is a technically feasible and safe operation and could achieve superior clinical outcomes compared with the multiportal approach for selected patients in specialized centers. We recommend learning uniportal thoracoscopic sleeve lobectomy in high-volume centers where surgeons can reach expert level in less time. Furthermore, large-scale multicenter randomized controlled studies are necessary to validate these results.

\section{Acknowledgments}

We sincerely thank all authors, sponsors and patients for their effort and participation in our included studies.

Funding: This work was supported by the Beijing Hope Marathon Special Fund of Chinese Cancer Foundation (LC2018L01).

\section{Footnote}

Reporting Checklist: The authors have completed the
STROBE reporting checklist. Available at http://dx.doi. org/10.21037/jtd-20-2695

Data Sharing Statement: Available at http://dx.doi. org/10.21037/jtd-20-2695

Peer Review File: Available at http://dx.doi.org/10.21037/jtd20-2695

Conflicts of Interest: All authors have completed the ICMJE uniform disclosure form (available at http://dx.doi. org/10.21037/jtd-20-2695). The authors have no conflicts of interest to declare.

Ethical Statement: The authors are accountable for all aspects of the work in ensuring that questions related to the accuracy or integrity of any part of the work are appropriately investigated and resolved. This study was conducted in accordance with the Declaration of Helsinki (as revised in 2013). Study had been approved by the Research Ethics Committee of Cancer Institute and Hospital of the Chinese Academy of Medical Sciences (No. 19-0851870) and informed consent was taken from all the patients. Patients' data was anonymized and maintained with confidentiality.

Open Access Statement: This is an Open Access article distributed in accordance with the Creative Commons Attribution-NonCommercial-NoDerivs 4.0 International License (CC BY-NC-ND 4.0), which permits the noncommercial replication and distribution of the article with the strict proviso that no changes or edits are made and the original work is properly cited (including links to both the formal publication through the relevant DOI and the license). See: https://creativecommons.org/licenses/by-nc-nd/4.0/.

\section{References}

1. Ludwig C, Stoelben E, Olschewski M, et al. Comparison of morbidity, 30-day mortality, and long-term survival after pneumonectomy and sleeve lobectomy for non-small cell lung carcinoma. Ann Thorac Surg 2005;79:968-73.

2. Santambrogio L, Cioffi U, De SM, et al. Video-assisted sleeve lobectomy for mucoepidermoid carcinoma of the left lower lobar bronchus: a case report. Chest 2002;121:635-6.

3. Yildizeli B, Fadel E, Mussot S, et al. Morbidity, mortality, and long-term survival after sleeve lobectomy for 
non-small cell lung cancer. Eur J Cardiothorac Surg 2007;31:95-102.

4. Mahtabifard A, Fuller CB, McKenna RJ Jr. Video-assisted thoracic surgery sleeve lobectomy: a case series. Ann Thorac Surg 2008;85:S729-32.

5. Gonzalez D, Paradela M, Garcia J, et al. Single-port videoassisted thoracoscopic lobectomy. Interact Cardiovasc Thorac Surg 2011;12:514-5.

6. Gonzalez-Rivas D, Fernandez R, Fieira E, et al. Uniportal video-assisted thoracoscopic bronchial sleeve lobectomy: first report. J Thorac Cardiovasc Surg 2013;145:1676-7.

7. Gonzalez-Rivas D, Delgado M, Fieira E, et al. Left lower sleeve lobectomy by uniportal video-assisted thoracoscopic approach. Interact Cardiovasc Thorac Surg 2014;18:237-9.

8. Guido W, Gonzalez-Rivas D, Duang L, et al. Uniportal video-assisted thoracoscopic right upper sleeve lobectomy. J Vis Surg 2015;1:10.

9. Gonzalez-Rivas D, Yang Y, Sekhniaidze D, et al. Uniportal video-assisted thoracoscopic bronchoplastic and carinal sleeve procedure. J Thorac Dis 2016;8:S210-22.

10. Yang C, Akar FA, Chen J, et al. Right sleeve pneumonectomy via uniportal video-assisted thoracoscopic approach. J Thorac Dis 2018;10:E391-6.

11. Gonzalez-Rivas D, Delgado M, Fieira E, et al. Double sleeve uniportal video-assisted thoracoscopic lobectomy for non-small cell lung cancer. Ann Cardiothorac Surg 2014;3:E2.

12. Gonzalez-Rivas D, Marin JC, Granados JP, et al. Uniportal video-assisted thoracoscopic right upper sleeve lobectomy and tracheoplasty in a 10 -year-old patient. J Thorac Dis 2016;8:E966-9.

13. Zhou S, Pei G, Han Y, et al. Sleeve lobectomy by videoassisted thoracic surgery versus thoracotomy for non-small cell lung cancer. J Cardiothorac Surg 2015;10:116.

14. Chen H, Xu G, Zheng B, et al. Initial experience of singleport video-assisted thoracoscopic surgery sleeve lobectomy and systematic mediastinal lymphadenectomy for nonsmall-cell lung cancer. J Thorac Dis 2016;8:2196-202.

15. Ma S, Yan T, Liu D, et al. Initial experience of sleeve lobectomy under complete video-assisted thoracic surgery. Asia Pac J Clin Oncol 2018;14:114-9.

16. Rami-Porta R, Asamura H, Travis WD, et al. Lung. In: Amin MB, Edge SB, Greene FL, et al. editors. AJCC Cancer Staging Manual. 8th ed. New York: Springer, 2017:431-56.

17. Zhang Y, Liu S, Han Y, et al. Robotic anatomical segmentectomy: an analysis of the learning curve. Ann Thorac Surg 2019;107:1515-22.

18. Koryllos A, Stoelben E. Uniportal video-assisted thoracoscopic surgery (VATS) sleeve resections for nonsmall cell lung cancer patients: an observational prospective study and technique analysis. J Vis Surg 2018;4:16.

19. Li Y, Wang J. Video-assisted thoracoscopic surgery sleeve lobectomy with bronchoplasty: an improved operative technique. Eur J Cardiothorac Surg 2013;44:1108-12.
Cite this article as: Zhao J, Zeng Q, Li J, Tan F, Xue Q, Mu J, Gao Y, Wang D, Gao S. Uniportal versus multiportal thoracoscopic sleeve lobectomy for the surgical treatment of centrally located lung cancer: a single institution experience. J Thorac Dis 2020;12(12):7145-7155. doi: 10.21037/jtd-20-2695 